Journal for ImmunoTherapy of Cancer

\section{JME-001 phase II trial of first-line combination chemotherapy with cisplatin, pemetrexed, and nivolumab for unresectable malignant pleural mesothelioma}

To cite: Miyamoto Y, Kozuki T, Aoe K, et al. JME-001 phase II trial of first-line combination chemotherapy with cisplatin, pemetrexed, and nivolumab for unresectable malignant pleural mesothelioma. Journal for ImmunoTherapy of Cancer 2021;11:e003288. doi:10.1136/ jitc-2021-003288

- Additional supplemental material is published online only. To view, please visit the journal online (http://dx.doi.org/10. 1136/jitc-2021-003288).

Accepted 07 October 2021
(C) Author(s) (or their employer(s)) 2021. Re-use permitted under CC BY. Published by BMJ.

${ }^{1}$ Department of Medical Oncology, Okayama Rosai Hospital, Okayama, Japan ${ }^{2}$ Department of Thoracic Oncology and Medicine, National Hospital Organization Shikoku Cancer Center, Matsuyama, Japan

${ }^{3}$ Department of Medical Oncology, National Hospital Organization Yamaguchi-Ube Medical Center, Ube, Japan

${ }^{4}$ Center of Innovative Clinical Medicine, Okayama University Hospital, Okayama, Japan

Correspondence to Dr Nobukazu Fujimoto; nobufujimot@gmail.com

\section{ABSTRACT}

Background JME-001 is a phase II trial assessing the efficacy and safety of cisplatin, pemetrexed, and nivolumab as first-line therapy in malignant pleural mesothelioma (MPM).

Patients and methods Patients with untreated, unresectable MPM with an Eastern Cooperative Oncology Group (ECOG) performance status (PS) of 0-1 were included. The primary endpoint is the centrally reviewed objective response rate. The secondary endpoints include (1) response rate assessed by investigators, (2) disease control rate, (3) overall survival, (4) progression-free survival, (5) duration of response, and (6) time to response. Safety and adverse events will also be evaluated. Cisplatin $(75 \mathrm{mg} /$ $\left.\mathrm{m}^{2}\right)$, pemetrexed $\left(500 \mathrm{mg} / \mathrm{m}^{2}\right)$, and nivolumab ( $360 \mathrm{mg} /$ body) were administered intravenously every 3 weeks with a total of 4-6 cycles. If patients did not progress during the combination phase, maintenance therapy with nivolumab was administered until disease progression or unacceptable toxicity. Tissue samples were required and collected for programmed death ligand 1 analysis.

Results Eighteen patients (mean age 69.2 years, 15 men) were enrolled between January 2018 and May 2019. The ECOG PS was 0 in 3 patients and 1 in 15 patients. Fourteen (77.8\%; $95 \% \mathrm{Cl} 52.4 \%$ to $93.6 \%$ ) patients had an objective response. The disease control rate was $94.4 \%(95 \% \mathrm{Cl}$ $72.7 \%$ to $99.9 \%$ ). Fourteen $(77.8 \%$ ) patients had partial response (PR), three had stable disease, and one was not evaluable. Tumor shrinkage was observed in 10/14 (71.4\%) patients with epithelioid, and 2/2 (100\%) patients with sarcomatoid or biphasic histological subtype had PR. Ten (55.6\%) patients experienced grade 3 or worse adverse events, including disorder of metabolism or nutrition (33.3\%), loss of appetite (27.8\%), anemia (16.7\%), and hyponatremia (11.1\%). No treatment-related deaths occurred.

Conclusions The safety and efficacy of this study strongly support a definitive trial of this combination.

Trial registration number

UMIN000030892.

\section{INTRODUCTION}

Malignant pleural mesothelioma (MPM) is an aggressive tumor that arises from mesothelial-lined surfaces and has a poor survival rate. ${ }^{1}$ The industrial use of asbestos has been banned in Japan since 2006, but the incidence of MPM is expected to continue to increase for the next few decades due to past usage of asbestos. ${ }^{2}$ Treatment of MPM is challenging. Most cases are diagnosed at an advanced stage and treated with systemic chemotherapy. Combination chemotherapy with cisplatin and pemetrexed is the standard treatment regimen; however, the median overall survival (OS) is only about 12 months. ${ }^{3}$ Recently, the addition of bevacizumab was shown to improve OS when added to cisplatin and pemetrexed in the treatment of unresectable MPM. ${ }^{4}$ However, the prolongation of OS was less than 3 months and it can only be administered to bevacizumabeligible patients. Therefore, cisplatin and pemetrexed is still considered the standard treatment regimen and additional treatment options are urgently needed.

Immune checkpoint inhibitors (ICIs), such as programmed death-1 (PD-1), programmed death-ligand 1 (PD-L1), and cytotoxic $\mathrm{T}$ lymphocyte associated protein-4 (CTLA4), have revolutionized cancer treatment. Nivolumab is a human monoclonal antibody that targets the PD-1 cluster of differentiation 279 cell surface membrane receptor. Binding of PD-1 to its ligands, PD-L1 and PD-L2, results in the downregulation of lymphocyte activation. Nivolumab inhibits the interaction between PD-1 and its ligands, promotes immune responses, and triggers antitumor activity and has already been approved in Japan for multiple types of cancer, including malignant melanoma, non-small cell lung cancer, and gastric cancer. Mesothelioma carcinogenesis occurs on the background 
of the chronic inflammatory responses to asbestos, and the tumor microenvironment is composed of proinflammatory cytokines, growth factors, endothelial cells, stromal cells, and immune cells. ${ }^{5}$ Thus, there is a strong biological rationale to use ICIs in MPM. A phase II trial has demonstrated a favorable response to nivolumab in previously treated MPM. ${ }^{6}$ Based on the results, nivolumab has been approved for patients with MPM that is refractory or intolerable to platinum/pemetrexed chemotherapy.

A recent report indicated that platinum drugs enhance the effector immune response through modulation of PD-L1. ${ }^{7}$ These encouraging results may extend to the first-line treatment of MPM with the hope of enhancing the antitumor response, particularly when used in combination with the current standard chemotherapy. Unfortunately, no prospective clinical trial is being conducted to evaluate the combination of nivolumab and cisplatin/ pemetrexed. Therefore, we launched the current trial to assess combination chemotherapy with cisplatin, pemetrexed, and nivolumab for MPM.

\section{MATERIALS AND METHODS Study design and patients}

JME-001 is a single-arm, prospective, non-randomized, non-comparative, open label, multicenter, phase II trial conducted from January 1, 2018, to November 30, 2019 (data cut-off date), at four centers in Japan. All patients who met the inclusion and exclusion criteria (online supplemental tables 1 and 2) were invited for screening. Eligible patients were $\geq 20$ years old with histologically confirmed, untreated, unresectable advanced MPM and had $\geq 1$ measurable lesion(s) as defined in the modified Response Evaluation Criteria in Solid Tumors V.1.1 (mRECIST) ${ }^{8}$ for mesothelioma and confirmed by imaging within 14 days prior to enrollment. Eligible patients also had to have tumor tissue samples available for the analysis of PD-L1 expression and an Eastern Cooperative Oncology Group (ECOG) performance status of 0 or 1. Main exclusion criteria were severe hypersensitivity reactions to any other drug, including antibody products; concurrent autoimmune disease or a history of chronic or recurrent autoimmune disease; multiple primary cancers; brain metastases; current or history of interstitial lung disease or pulmonary fibrosis diagnosed based on imaging or clinical findings; or previous treatment with nivolumab, anti-PD-1 antibody, anti-PD-L1 or PD-L2, or any other therapeutic antibodies or pharmacotherapies for T-cell regulation.

\section{Procedures}

Treatment comprised two sequential phases: the combination phase and the maintenance phase. In the combination phase, cisplatin $\left(75 \mathrm{mg} / \mathrm{m}^{2}\right)$, pemetrexed $(500$ $\left.\mathrm{mg} / \mathrm{m}^{2}\right)$, and nivolumab (360 mg/body) were administered intravenously. Nivolumab was kindly provided by Ono Pharmaceutical. This treatment was mandated to repeat every 3 weeks for a total of 4-6 cycles. If there was no progression of MPM during the combination phase, maintenance therapy with nivolumab was administered until disease progression, unacceptable toxicity, or the patient's condition met the withdrawal criteria.

Both cisplatin and pemetrexed are usually administered every 3 weeks. Under the consideration of practical utility and dose intensity, we planned to administer nivolumab every 3 weeks at the dose of $360 \mathrm{mg} /$ body. Patients underwent tumor imaging by CT or MRI every three cycles. Target lesion diameters were measured, and the tumor response was assessed according to mRECIST criteria.

PD-L1 expression was analyzed in a central laboratory (Cancer Genetics, New Jersey, USA) using archival tumor tissue samples with 28-8 antibody (Dako, California). One or more formalin-fixed, paraffin-embedded (FFPE) blocks of tumor tissue samples collected by core needle biopsy, excisional biopsy, or incisional biopsy of $\geq 5 \mathrm{FFPE}$ unstained slide samples (serial tissue sections) were analyzed for PD-L1 status. Each sample was required to contain $\geq 100$ evaluable tumor cells. PD-L1-positive was defined as membranous staining in $\geq 1 \%$ of tumor cells. Samples were classified as not evaluable (NE) if the biological conditions of the sample rendered the stained cell membranes difficult to assess, even if the samples otherwise met the evaluation criteria.

\section{Outcomes}

This study assessed the efficacy and safety of first-line combination therapy with cisplatin, pemetrexed, and nivolumab for advanced or metastatic MPM. The primary endpoint was the centrally assessed objective response according to mRECIST. The objective response rate (ORR) was defined as the proportion of patients whose best overall response was a complete response (CR) or partial response (PR). The secondary endpoints included efficacy evaluated by the (1) response rate assessed by investigators, (2) disease control rate, (3) OS, (4) progression-free survival (PFS), (5) response duration, and (6) time to response. Safety and adverse events were also evaluated.

The OS was defined as the duration from study registration until the date of death from any cause. PFS was defined as the time from registration to first progressive disease (PD) or death from any cause, whichever is earlier. The disease control rate was the percentage of patients whose best overall response was CR, PR, or stable disease (SD).

Adverse events (AEs) and treatment-related AEs (TRAEs) were monitored throughout the study period and graded according to the National Cancer Institute Common Terminology Criteria for Adverse Events, V.4.0. Quality of life (QOL) was evaluated using the EuroQol 5 Dimension Japanese edition ${ }^{9}$ and the Lung Cancer Symptom Scale for Mesothelioma. ${ }^{10}$ QOL was evaluated at each treatment visit according to the treatment schedule before the administration of agents. 


\section{Statistical analysis}

The trial size was set as 18 due to feasibility. If we assume that 6-12 patients would have a response, the response rate would be $33.3 \%-66.7 \%$. In this case, the estimate accuracy indicates that the range between the point estimate of the response rate and the lower confidence limit (two-sided 95\% confidence coefficient based on exact test) would be $18 \%-22 \%$.

The statistical analysis was conducted based on predetermined statistical analysis plan. The efficacy and safetyrelated endpoints were analyzed with full and safety analysis sets, respectively. The patient characteristics, the numbers of treatment cycles and dose reductions, duration of treatment, the relative dose intensity and trial continue/discontinue condition with the reasons were summarized. The centrally reviewed ORR (primary endpoint), investigator-assessed ORR and the disease control rate (included in the secondary endpoints) were estimated with 95\% CI. Response rate per histological subtypes and PD-L1 expression status were also calculated. The other secondary endpoints; OS, PFS, duration of response, and time to response were analyzed based on the Kaplan-Meier product limit approach. The best reduction percentage and the change in the sum of target lesions from baseline in each patient were graphed (waterfall and spider plots). The frequency of AEs and TRAEs were summarized with the grade. The summary statistics of the QOL scale/score difference between time points was calculated.

\section{Role of the funding source}

The funding source was not involved in the study design, the collection, analysis, and interpretation of data, writing the report, or in the decision to submit the paper for publication.

\section{RESULTS}

Eighteen patients were enrolled between January 2018 and May 2019 (table 1). Four patients (22.2\%) continued treatment, and $14(77.8 \%)$ discontinued treatment until data cut-off (November 30, 2019). The reasons for discontinuation included PD ( $\mathrm{n}=8,44.4 \%)$, development of a grade 3 or greater infusion reaction $(n=1,5.6 \%)$, and continuation of treatment judged as inappropriate by the principal investigator $(n=3,16.7 \%)$. One patient withdrew consent to the treatment after the first cycle of induction chemotherapy. All 18 patients were included in both the full and safety analysis sets. Median follow-up was 15.2 (range 6.9-19.4) months.

Patients received an average of 4.8 (range 2-6) cycles of induction triplet chemotherapy. Nine patients $(50.0 \%)$ received four cycles and eight patients $(44.4 \%)$ received six cycles. The average number of dose reductions was 0 for nivolumab, 0.3 (range $0-1$ ) for pemetrexed, and 0.3 (range $0-1$ ) for cisplatin. The relative dose intensity in combination phase was $93.5 \%$ (range $75.0 \%-$ $100.0 \%$ ) for nivolumab, $89.4 \%$ (range $60.9 \%-101.3 \%$ )

\begin{tabular}{|c|c|}
\hline Characteristic & Value \\
\hline Median age, years (range) & $69(64-78)$ \\
\hline Male/female & 15 (83) / 3 (17) \\
\hline ECOG PS, 0/1 & $3(17) / 15(83)$ \\
\hline \multicolumn{2}{|l|}{ Histological subtype } \\
\hline Epithelioid & $14(77.8)$ \\
\hline Sarcomatoid & $2(11.1)$ \\
\hline Biphasic & $2(11.1)$ \\
\hline \multicolumn{2}{|l|}{ TNM classification } \\
\hline T1NOMO & $2(11.1)$ \\
\hline T1N2M0 & $1(5.6)$ \\
\hline T2NOMO & $1(5.6)$ \\
\hline T3NOMO & $6(33.3)$ \\
\hline T3N2M0 & $1(5.6)$ \\
\hline T4NOMO & $3(16.7)$ \\
\hline T4N1M0 & $1(5.6)$ \\
\hline T4N2M0 & $2(11.1)$ \\
\hline T4N2M1 & $1(5.6)$ \\
\hline \multicolumn{2}{|l|}{ Stage } \\
\hline 1 & $8(44.4)$ \\
\hline II & $0(0.0)$ \\
\hline III & $9(50)$ \\
\hline IV & $1(5.6)$ \\
\hline \multicolumn{2}{|l|}{ PD-L1 expression } \\
\hline$<1 \%$ & $1(5.6)$ \\
\hline$\geq 1 \%$ & $17(94.4)$ \\
\hline
\end{tabular}

Values are $n(\%)$ unless otherwise noted.

ECOG, Eastern Cooperative Oncology Group; PD-L1, programmed death-ligand 1; PS, performance status; TNM, tumor, node, metastases.

for pemetrexed, and $90.1 \%$ (range $63.8 \%-101.1 \%$ ) for cisplatin. The average number of nivolumab total cycles was 10.9 (range 2-26). The average total duration of treatment was 7.5 (range $0.7-18.7$ ) months.

The best percentage reduction and the change in the sum of target lesions in each patient are shown in figure 1. Fourteen (77.8\%; 95\% CI, 52.4\% to $93.6 \%$ ) patients had an objective response by central assessment (table 2), which was consistent with the investigator-assessed objective response. Regarding best overall response, 14 patients had a PR. The responses and disease control rate are given in table 2. Tumor shrinkage was observed in all histological subtypes, in 10/14 (71.4\%) patients with epithelioid, and the four patients with non-epithelioid disease had a PR. The three remaining patients with epithelioid had SD and one remaining patient with epithelioid was NE. Tumor shrinkage was observed regardless of PD-L1 status and occurred in 13/17 (76.5\%) patients with PD-L1 

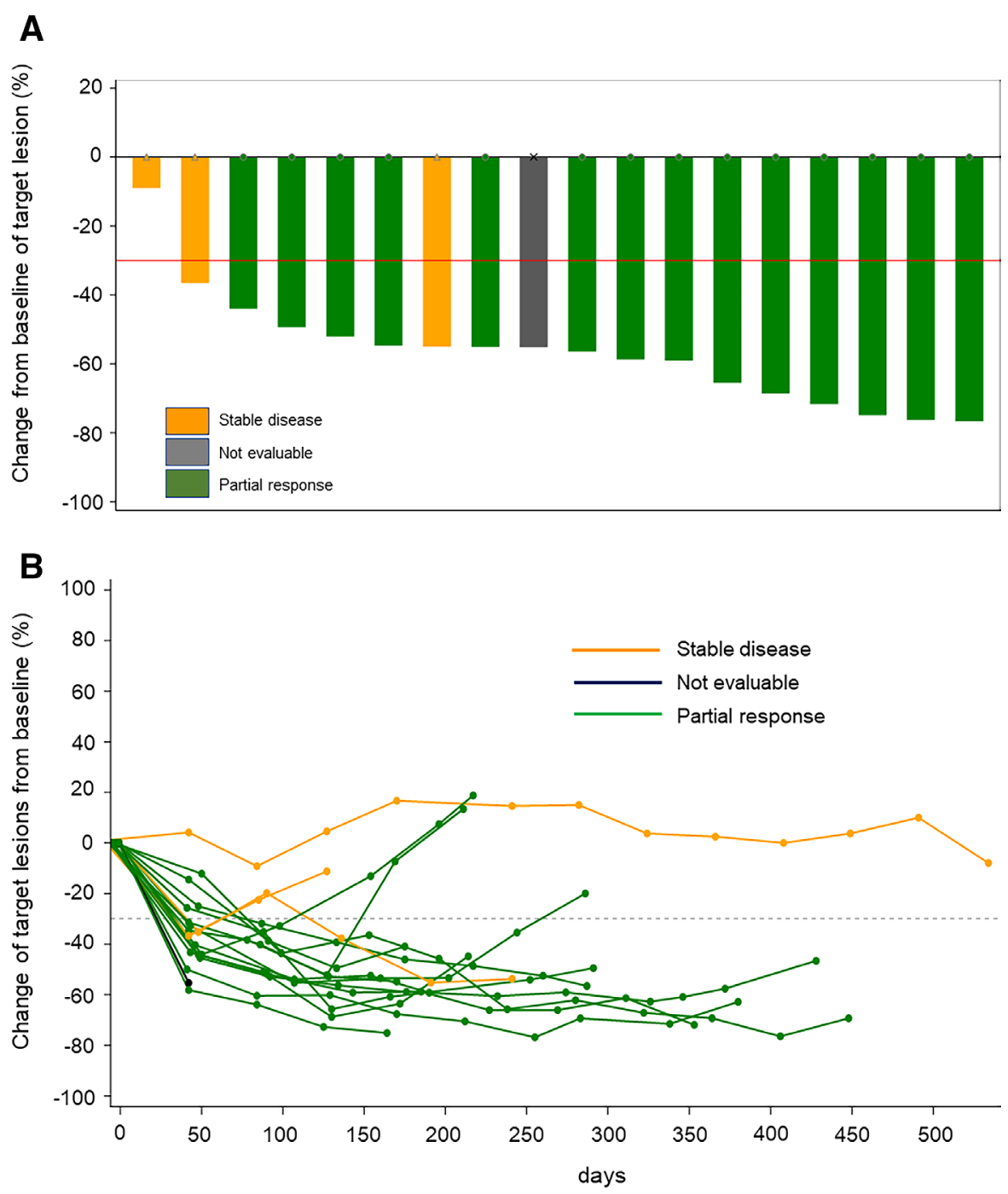

Figure 1 The best reduction percentage $(A)$ and the change in the of sum of target lesions $(B)$ in each patient.

expression $\geq 1 \%$ and $1 / 1$ (100\%) patients with PD-L1 expression $<1 \%$.

At data cut-off, three patients $(16.7 \%)$ had an ongoing response. The median response duration was 6.7 months (95\% CI 4.21 to not reached), with median time to response of 1.54 (range 1.4-3.3) months. The median

\begin{tabular}{|c|c|c|}
\hline & No. of patients & $\%$ \\
\hline \multicolumn{3}{|c|}{ Response } \\
\hline $\mathrm{CR}$ & 0 & 0 \\
\hline PR & 14 & 77.8 \\
\hline SD & 3 & 16.7 \\
\hline PD & 0 & 0 \\
\hline NE & 1 & 5.6 \\
\hline \multicolumn{2}{|c|}{ Response rate $(95 \% \mathrm{Cl})$} & 77.8 (52.4 to 93.6 ) \\
\hline \multicolumn{2}{|c|}{ Disease control rate $(95 \% \mathrm{Cl})$} & 94.4 (72.7 to 99.9$)$ \\
\hline
\end{tabular}

$\mathrm{CR}$, complete response; NE, not evaluable; PD, progressive disease; PR, partial response; SD, stable disease. reduction in target lesions from baseline (depth of response) was $55.9 \%$ (IQR 52.2\%-68.8\%).

The Kaplan-Meier curve for PFS, determined by blinded independent central review, is shown in online supplemental figure 1A. At the time of data cut-off, 11 $(61.1 \%)$ events had occurred, and $7(38.9 \%)$ cases were censored. All 11 events were PD, and there was no death before disease progression. Median PFS was 8.02 months (95\% CI 5.59 to 14.06). The 6-month and 12-month PFS rate was $69.0 \%$ (95\% CI $40.8 \%$ to $85.8 \%$ ) and $40.3 \%(95 \%$ CI $16.2 \%$ to $63.5 \%$ ), respectively. The Kaplan-Meier curve for OS is shown in online supplemental figure 1B. At the time of data cut-off, 2 (11.1\%) events had occurred, and $16(88.9 \%)$ cases were censored. Median OS was 20.8 months. The 6-month and 12-month overall survival rate was $100 \%$ (95\% CI $100.0 \%$ to $100.0 \%$ ) and $92.3 \%(95 \%$ CI $56.6 \%$ to $98.9 \%$ ), respectively.

All 18 patients experienced AEs, but no treatmentrelated death was recorded. All-cause AEs occurring in $\geq 10 \%$ of patients are shown in table 3 . Ten $(55.6 \%)$ patients experienced grade 3 or greater AEs, including disorder of metabolism or nutrition, loss of appetite, 


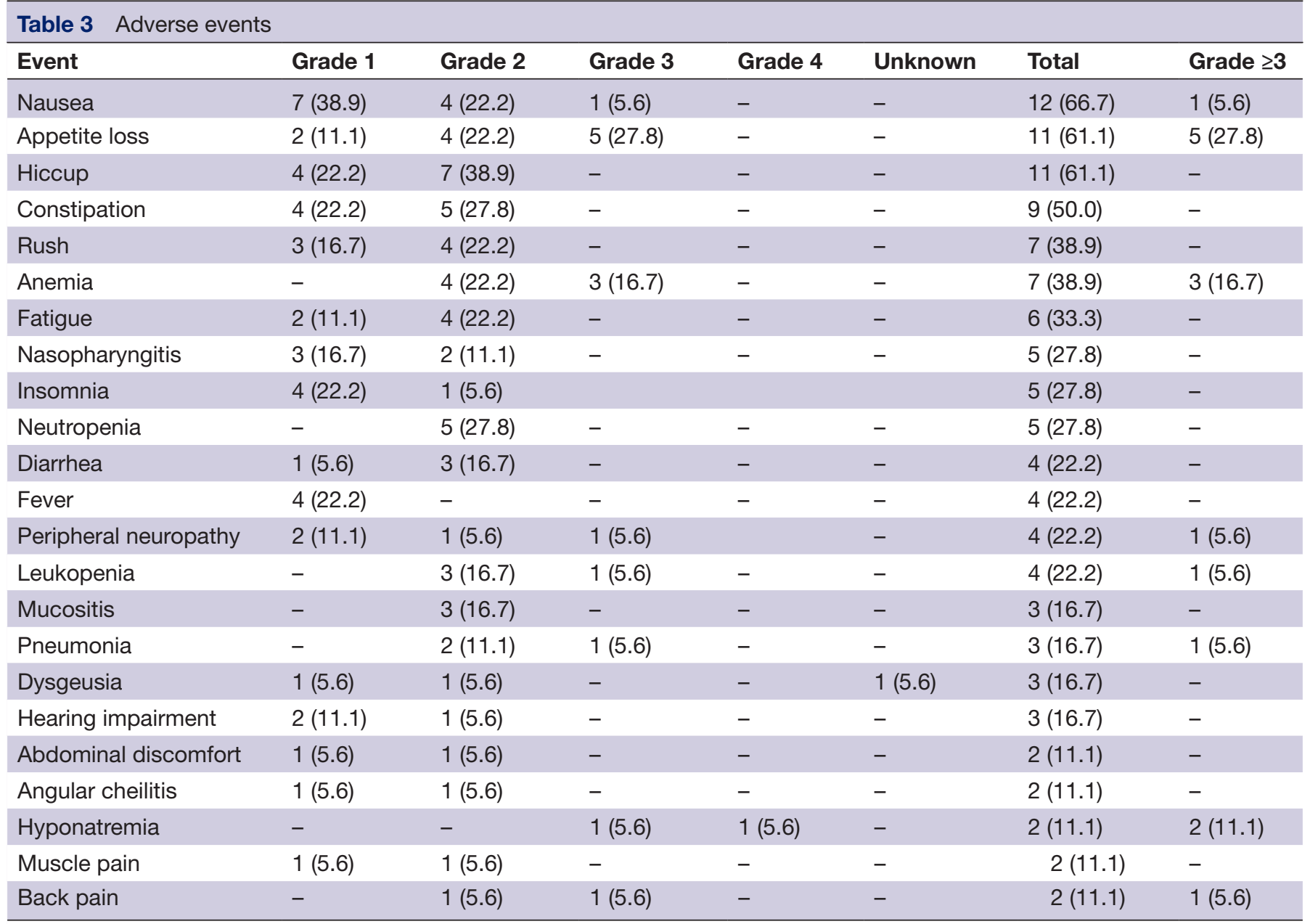

Values are $\mathrm{n}(\%)$.

anemia, hyponatremia, leukopenia, lymphocytopenia, increased serum alanine aminotransferase, increased serum aspartate aminotransferase, pneumonia, nausea, colitis, diverticulitis, dental pulpitis, pulmonary embolism, peripheral neuropathy, and back pain. Two patients (11.1\%) experienced peripheral neuropathy during nivolumab maintenance treatment, leading to treatment discontinuation.

The mean $( \pm \mathrm{SD})$ difference in the health visual analog scale based on the start of the induction treatment was $-5.6 \pm 24.2 \mathrm{~mm}$ (range -65 to 30 ) at the start of nivolumab maintenance treatment and $0.5 \pm 23.3 \mathrm{~mm}$ (range -40 to 30 ) at the end of the treatment. The mean $( \pm \mathrm{SD})$ difference in the index score based on the start of the induction treatment was $0.0185 \pm 0.1389$ (range -0.319 to 0.292 ) at the start of nivolumab maintenance treatment and $-0.0166 \pm 0.1912(-0.364$ to 0.292$)$ at the end of the treatment. The mean $( \pm \mathrm{SD})$ difference in the total visual analog scale based on the start of the induction treatment was $-0.01 \pm 13.57 \mathrm{~mm}(-21.1$ to 28.1) at the start of nivolumab maintenance treatment and $-2.11 \pm 21.38 \mathrm{~mm}(-41.3$ to 36.0$)$ at the end of the treatment.

\section{DISCUSSION}

To the best of our knowledge, this study is the first clinical trial to evaluate the effect of combining nivolumab and platinum-based chemotherapy for the treatment of advanced MPM. The combination of an ICI and cytotoxic chemotherapy is a rapidly evolving area of interest in cancer treatment. Cytotoxic agents, including platinum, could modulate the immune response through PD-1/ PD-L1 inhibition by enhancing the potential immunogenic effect. ${ }^{11-13}$ Combination regimens that include a PD-1 or PD-L1 inhibitor have led to prolonged OS in small cell lung cancer ${ }^{14}$ and non-small cell lung cancer. ${ }^{15}$ Previous reports have also shown that cytotoxic agents can induce immune-stimulating properties in mesothelioma cell models. ${ }^{1617}$

Nivolumab is currently administered at a dose of $240 \mathrm{mg} /$ body biweekly in clinical practice based on recent clinical trials. ${ }^{6}{ }^{18}$ However, combination chemotherapy with cisplatin and pemetrexed is administered every 3 weeks. In the current study, nivolumab was administered every 3 weeks at a dose of $360 \mathrm{mg} /$ body based on a recent report that the combination of nivolumab $(10 \mathrm{mg} / \mathrm{kg})$ and pemetrexed/cisplatin every 3 weeks has 
an acceptable toxicity profile and encouraging antitumor activity in patients with advanced non-small cell lung cancer. $^{19}$

We set a centrally assessed ORR according to mRECIST as the primary endpoint. A modification of the RECIST criteria has specifically addressed the difficulties measuring and assessing changes in tumor bulk in MPM. In addition, the mRECIST criteria have successfully distinguished between responders and non-responders for the parameters of OS, ${ }^{20}$ demonstrating its ability as an appropriate endpoint, particularly in phase II studies. The combination of nivolumab and cisplatin/pemetrexed has demonstrated a notable ORR of $77.8 \%$. This is the highest ORR reported thus far in chemotherapy for MPM. Moreover, all participants demonstrated tumor shrinkage. One of the most remarkable aspects of the participants in the current study was a high tumor proportion score for PD-L1 expression. PD-L1 is expressed in a substantial proportion of MPM and is associated with poor survival. ${ }^{21}$ The association of PD-L1 expression in mesothelioma cells and the response to anti-PD-1 inhibitors are still controversial. PD-L1 positivity was not correlated with outcome in one trial, ${ }^{22}$ but increased ORR and prolonged survival was observed in patients with PD-L1-positive patients in another study. ${ }^{6}$ Nivolumab plus ipilimumab combination therapy exhibited higher ORR in patients with PD-L1-positive MPM compared with that in patients with PD-L1-negative MPM. ${ }^{23}$ In another study, PD-L1 expression was not only associated with the increase of ORR but also associated with the improvement in PFS and OS when treated with a combination of nivolumab plus ipilimumab. ${ }^{24}$ These results indicate that PD-L1 expression could be a reliable biomarker for ICI response. The high PD-L1 expression may contribute to the favorable response in the current study. The $\mathrm{AE}$ profile in the current study was consistent with what is expected when combining cisplatin and pemetrexed with nivolumab. The addition of nivolumab did not appear to increase the frequency or severity of AEs associated with chemotherapy with cisplatin and pemetrexed.

Recently, a multicenter phase II study was conducted in Australia ${ }^{25}$ in 55 patients with untreated MPM who received cisplatin, pemetrexed, and durvalumab for a maximum of six cycles, followed by durvalumab maintenance for up to 12 months. The primary endpoint, 6-month PFS, was 57\%, and the ORR and disease control rate were $48 \%$ and $87 \%$, respectively. Based on these favorable results, a multicenter trial is planned to randomize participants for cisplatin and pemetrexed with or without durvalumab. More recently, an international randomized phase III trial evaluated the combination of ipilimumab, a CTLA-4 inhibitor, and nivolumab versus standard firstline platinum-pemetrexed chemotherapy in treatmentnaïve patients with untreated, unresectable MPM. ${ }^{26}$ The primary endpoint of OS was met with a 4-month prolongation in median OS in those who received nivolumab-ipilimumab compared with those who received platinum-pemetrexed chemotherapy. These findings led to the recent approval of nivolumab plus ipilimumab in the USA for first-line treatment of unresectable MPM. The combination of nivolumab and ipilimumab would be a new standard first-line treatment, but some problems still remain. One of the problems is a rapid drop-off in PFS in patients receiving nivolumab plus ipilimumab. Similar results have been shown in clinical trials of nonsmall cell lung cancer, which has shown improvement in OS and PFS. ${ }^{27}$ A recent study of non-small cell lung cancer that ipilimumab plus nivolumab with two cycles cytotoxic chemotherapy demonstrated an improvement in the rapid drop-off of PFS and OS. ${ }^{28}$ These results support the further clinical development of the ICI-chemotherapy combination in first-line treatment of MPM.

The main limitation of the current study is its single-arm, non-comparative design. In addition, we included a few participants without tumor PD-L1 expression. Survival analyses are immature because most of the participants were censored at the time of data cut-off. The trial size was determined based not on statistical power, but on our ability to accrue patient. However, the estimated lower limit of the ORR in the current study was $52.4 \%$, which is higher than the ORRs reported in previous studies of front-line cisplatin/pemetrexed combination chemotherapy.

In conclusion, the combination of cisplatin, pemetrexed, and nivolumab demonstrated sufficient activity and safety as first-line therapy in unresectable MPM. We think that adding nivolumab to cisplatin/pemetrexed would be a treatment option for patients with advanced MPM, though the efficacy and safety should be examined in a definitive randomized study.

Contributors YM, TK, KA, DH, and SW were involved in the acquisition of the data. MY was involved in analysis of the data. YM, TK, KA, JS, KH, and NF were involved in the interpretation of the data, writing or reviewing and editing the manuscript. NF approved the final version of the manuscript for submission. NF is responsible for the overall content as guarantor.

Funding This work was supported by Ono Pharmaceutical and the Ministry of Health, Labor, and Welfare, Japan (grant number 180101-02).

Competing interests TK reports personal fees from Eli Lilly Japan, personal fees from Bristol Myers Squibb, personal fees from Ono Pharmaceutical, during the conduct of the study; grants and personal fees from Taiho Pharmaceutical, grants and personal fees from Kyowa Hakko Kirin, personal fees from AstraZeneca, personal fees from MSD, personal fees from Chugai Pharmaceutical, personal fees from Nippon Boehringer Ingelheim, personal fees from Merck Biopharma, personal fees from Nippon Kayaku, personal fees from Daiichi Sankyo, personal fees from Pfizer Japan, personal fees from Takeda Pharmaceutical, personal fees from Novartis, outside the submitted work. DH reports personal fees from Eli Lilly Japan, grants and personal fees from Bristol Myers Squibb, grants and personal fees from Ono Pharmaceutical, during the conduct of the study; personal fees from Kyowa Hakko Kirin, grants and personal fees from AstraZeneca, personal fees from Nippon Boehringer Ingelheim, grants and personal fees from MSD, personal fees from Taiho Pharmaceutical, grants and personal fees from Chugai Pharmaceutical, grants from Novartis, grants from Kissei Pharmaceutical, grants from Takeda Pharmaceutical, grants from Pfizer Japan, outside the submitted work. KA reports grants and personal fees from Eli Lilly Japan, grants and personal fees from Bristol Myers Squibb, grants and personal fees from Ono Pharmaceutical, during the conduct of the study; grants and personal fees from AstraZeneca, personal fees from Nippon Boehringer Ingelheim, grants and personal fees from MSD, grants from Novartis, outside the submitted work. KH reports grants from Bristol Meyers Squibb, MSD, AstraZeneca, Chugai and Eli Lilly Japan, and honoraria from Eli Lilly Japan, Bristol Meyers Squib, Ono, Pfizer, AstraZeneca, Chugai, Takeda, MSD, Nippon Kayaku, 
Taiho, and Boehringer Ingelheim. NF reports personal fees from Eli Lilly Japan, grants, personal fees and other from Bristol Myers Squibb, grants, personal fees and other from Ono Pharmaceutical, during the conduct of the study; grants from MSD, personal fees from Chugai, personal fees from Daiichi Sankyo, outside the submitted work. Other authors have stated that they have no conflicts of interest.

\section{Patient consent for publication Not applicable.}

Ethics approval JME-001 was conducted in accordance with the Declaration of Helsinki and Good Clinical Practice Guidelines in Japan. The study protocol was reviewed and approved by the institutional review board of each site before study initiation. All patients provided written informed consent.

Provenance and peer review Not commissioned; externally peer reviewed.

Data availability statement Data are available upon reasonable request. The patients' de-identified clinical data may be made available to other investigators after approval by the institutional review board. Requests should be directed to the corresponding author.

Supplemental material This content has been supplied by the author(s). It has not been vetted by BMJ Publishing Group Limited (BMJ) and may not have been peer-reviewed. Any opinions or recommendations discussed are solely those of the author(s) and are not endorsed by BMJ. BMJ disclaims all liability and responsibility arising from any reliance placed on the content. Where the content includes any translated material, BMJ does not warrant the accuracy and reliability of the translations (including but not limited to local regulations, clinical guidelines, terminology, drug names and drug dosages), and is not responsible for any error and/or omissions arising from translation and adaptation or otherwise.

Open access This is an open access article distributed in accordance with the Creative Commons Attribution 4.0 Unported (CC BY 4.0) license, which permits others to copy, redistribute, remix, transform and build upon this work for any purpose, provided the original work is properly cited, a link to the licence is given, and indication of whether changes were made. See https://creativecommons.org/ licenses/by/4.0/.

\section{ORCID iD}

Nobukazu Fujimoto http://orcid.org/0000-0002-4516-0433

\section{REFERENCES}

1 Gemba K, Fujimoto N, Aoe K, et al. Treatment and survival analyses of malignant mesothelioma in Japan. Acta Oncol 2013;52:803-8.

2 Robinson BWS, Lake RA. Advances in malignant mesothelioma. $N$ Engl J Med 2005;353:1591-603.

3 Vogelzang NJ, Rusthoven JJ, Symanowski J, et al. Phase III study of pemetrexed in combination with cisplatin versus cisplatin alone in patients with malignant pleural mesothelioma. $J$ Clin Oncol 2003;21:2636-44.

4 Zalcman G, Mazieres J, Margery J, et al. Bevacizumab for newly diagnosed pleural mesothelioma in the mesothelioma avastin cisplatin pemetrexed study (maps): a randomised, controlled, openlabel, phase 3 trial. Lancet 2016;387:1405-14.

5 Ujiie H, Kadota K, Nitadori J-I, et al. The tumoral and stromal immune microenvironment in malignant pleural mesothelioma: a comprehensive analysis reveals prognostic immune markers. Oncoimmunology 2015;4:e1009285.

6 Okada M, Kijima T, Aoe K, et al. Clinical efficacy and safety of nivolumab: results of a multicenter, open-label, single-arm, Japanese phase II study in malignant pleural mesothelioma (merit). Clin Cancer Res 2019;25:5485-92.

7 Hato SV, Khong A, de Vries IJM, et al. Molecular pathways: the immunogenic effects of platinum-based chemotherapeutics. Clin Cancer Res 2014;20:2831-7.

8 Armato SG, Nowak AK. Revised modified response evaluation criteria in solid tumors for assessment of response in malignant pleural mesothelioma (version 1.1). J Thorac Oncol 2018;13:1012-21.
9 Tsuchiya A, lkeda S, Ikegami N, et al. Estimating an EQ5D population value set: the case of Japan. Health Econ 2002;11:341-53.

10 Hollen PJ, Gralla RJ, Liepa AM, et al. Adapting the lung cancer symptom scale (LCSS) to mesothelioma: using the LCSS-Meso conceptual model for validation. Cancer 2004;101:587-95.

11 Bracci L, Schiavoni G, Sistigu A, et al. Immune-based mechanisms of cytotoxic chemotherapy: implications for the design of novel and rationale-based combined treatments against cancer. Cell Death Differ 2014;21:15-25.

12 Wang Z, Till B, Gao Q. Chemotherapeutic agent-mediated elimination of myeloid-derived suppressor cells. Oncoimmunology 2017;6:e1331807.

13 Roselli M, Cereda V, di Bari MG, et al. Effects of conventional therapeutic interventions on the number and function of regulatory $T$ cells. Oncoimmunology 2013;2:e27025.

14 Horn L, Mansfield AS, Szczęsna A, et al. First-line Atezolizumab plus chemotherapy in extensive-stage small-cell lung cancer. $N$ Engl J Med 2018;379:2220-9.

15 Gandhi L, Rodríguez-Abreu D, Gadgeel S, et al. Pembrolizumab plus chemotherapy in metastatic non-small-cell lung cancer. $N$ Engl J Med 2018;378:2078-92.

16 Nowak AK, Lake RA, Marzo AL, et al. Induction of tumor cell apoptosis in vivo increases tumor antigen cross-presentation, crosspriming rather than cross-tolerizing host tumor-specific CD8 T cells. $\mathrm{J}$ Immunol 2003:170:4905-13.

17 Nowak AK, Robinson BWS, Lake RA. Synergy between chemotherapy and immunotherapy in the treatment of established murine solid tumors. Cancer Res 2003;63:4490-6.

18 Zhao X, Suryawanshi S, Hruska M, et al. Assessment of nivolumab benefit-risk profile of a $240-\mathrm{mg}$ flat dose relative to a $3-\mathrm{mg} / \mathrm{kg}$ dosing regimen in patients with advanced tumors. Ann Oncol 2017;28:2002-8.

19 Kanda S, Goto K, Shiraishi H, et al. Safety and efficacy of nivolumab and standard chemotherapy drug combination in patients with advanced non-small-cell lung cancer: a four arms phase lb study. Ann Oncol 2016;27:2242-50.

20 Byrne MJ, Nowak AK. Modified recist criteria for assessment of response in malignant pleural mesothelioma. Ann Oncol 2004;15:257-60.

21 Mansfield AS, Roden AC, Peikert T, et al. B7-H1 expression in malignant pleural mesothelioma is associated with sarcomatoid histology and poor prognosis. J Thorac Oncol 2014;9:1036-40.

22 Quispel-Janssen J, van der Noort V, de Vries JF, et al. Programmed death 1 blockade with nivolumab in patients with recurrent malignant pleural mesothelioma. $J$ Thorac Oncol 2018;13:1569-76.

23 Scherpereel A, Mazieres J, Greillier L, et al. Nivolumab or nivolumab plus ipilimumab in patients with relapsed malignant pleural mesothelioma (IFCT-1501 MAPS2): a multicentre, openlabel, randomised, non-comparative, phase 2 trial. Lancet Oncol 2019;20:239-53.

24 Disselhorst MJ, Quispel-Janssen J, Lalezari F, et al. Ipilimumab and nivolumab in the treatment of recurrent malignant pleural mesothelioma (initiate): results of a prospective, single-arm, phase 2 trial. Lancet Respir Med 2019;7:260-70.

25 Nowak AK, Lesterhuis WJ, Kok P-S, et al. Durvalumab with first-line chemotherapy in previously untreated malignant pleural mesothelioma (dream): a multicentre, single-arm, phase 2 trial with a safety run-in. Lancet Oncol 2020;21:1213-23.

26 Baas P, Scherpereel A, Nowak AK, et al. First-line nivolumab plus ipilimumab in unresectable malignant pleural mesothelioma (CheckMate 743): a multicentre, randomised, open-label, phase 3 trial. Lancet 2021;397:375-86.

27 Hellmann MD, Paz-Ares L, Bernabe Caro R, et al. Nivolumab plus ipilimumab in advanced non-small-cell lung cancer. N Engl J Med 2019;381:2020-31.

28 Paz-Ares L, Ciuleanu T-E, Cobo M, et al. First-line nivolumab plus ipilimumab combined with two cycles of chemotherapy in patients with non-small-cell lung cancer (CheckMate 9LA): an international, randomised, open-label, phase 3 trial. Lancet Oncol 2021;22:198-211. 